Case Report

\title{
Dorsal Dislocation of the Intermediate Cuneiform with a Medial Cuneiform Fracture: A Case Report and Review of the Literature
}

\author{
Burak Akan ${ }^{1,2}$ and Tugrul Yildirim ${ }^{1}$ \\ ${ }^{1}$ Department of Orthopedics and Traumatology, Faculty of Medicine, Ufuk University, Ankara, Turkey \\ ${ }^{2}$ Dikmen C Parkpinar Evleri, 9/B No. 28 Keklikpinari Cankaya, 06450 Ankara, Turkey \\ Correspondence should be addressed to Burak Akan; burakakan1977@yahoo.co.uk
}

Received 1 August 2013; Accepted 4 September 2013

Academic Editors: M. Gotoh, J. Mayr, and A. Saw

Copyright (c) 2013 B. Akan and T. Yildirim. This is an open access article distributed under the Creative Commons Attribution License, which permits unrestricted use, distribution, and reproduction in any medium, provided the original work is properly cited.

Dorsal dislocation of the intermediate cuneiform and isolated medial cuneiform fractures are rare injuries. In this report, we present a patient who sustained a dislocation of the intermediate cuneiform and describe predisposing factors and the treatment procedure.

\section{Introduction}

Dorsal dislocation of the intermediate cuneiform is a rare injury, and only a few cases have been reported [1-3]. The intermediate cuneiform is wedge shaped, lies between the medial and lateral cuneiforms, and is strongly attached to the first metatarsal. It is recessed at the second metatarsal base and forms the "keystone" of the Lisfranc tarsometatarsal joint complex [1]. Because it is wedge shaped and positioned dorsally, it has a tendency to dislocate dorsally, particularly when a plantar flexion force is applied to the midfoot [4]. Isolated cuneiform fractures are rarely observed and represent $1.7 \%$ of all tarsal fractures [5]. We present a case of a dorsally dislocated intermediate cuneiform and a fracture at the medial cuneiform. To our knowledge, dorsal dislocation of the intermediate cuneiform with a medial cuneiform fracture has not been previously reported in the literature.

\section{Case Report}

A 30-year-old woman sustained an injury to her right foot when she was walking in high-heeled shoes and fell down the stairs with her foot in an equinus and inversion position. The patient complained of severe pain and was unable to bear weight in her right foot. The initial clinical examination of her foot revealed swelling and tenderness at the dorsum of the midfoot without an open wound. There was no vascular compromise, and sensation was preserved. Plain radiographs showed dorsal dislocation of the intermediate cuneiform bone and a nondisplaced fracture at the medial cuneiform (Figure 1). A computed tomographic scan with threedimensional reconstruction supported the radiographical findings (Figure 2). The patient was taken to the operation room, and a closed reduction was attempted under general anesthesia, but it did not succeed. The dorsal longitudinal approach was then performed. Open reduction was performed with fluoroscopic control, and two $3.5 \mathrm{~mm}$ cortical lag screws were used for stabilization. The postoperative radiographs were satisfactory (Figure 3). A short leg posterior splint in the neutral plantigrade position was applied for three weeks. The active range of ankle motion started 3 weeks after surgery. The patient was advised to avoid weight-bearing activities for 6 weeks. At 3 months after surgery, plain radiographs revealed fusion of the medial cuneiform and no recurrence of the intermediate cuneiform dislocation. At the final follow-up, the patient had painless foot with normal range of motion. Screws were removed 12 months after surgery at the patient's own request.

\section{Discussion}

The three cuneiforms are wedge shaped and sit in the middle of the medial column of the foot. They are part of the transverse and medial longitudinal arches of the foot. The intermediate cuneiform is the smallest. Each cuneiform 


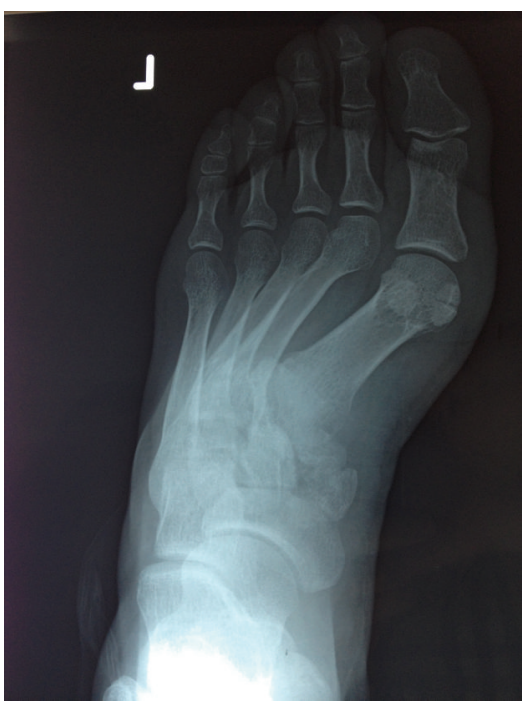

(a)

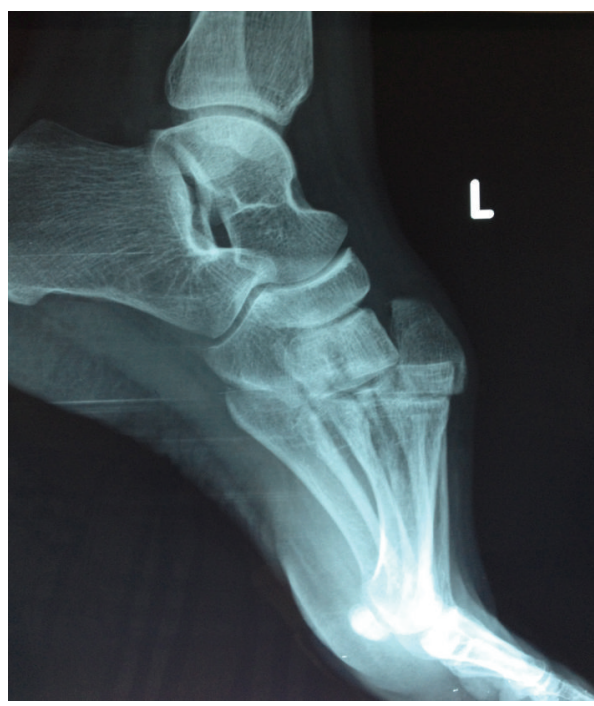

(b)

Figure 1: (a) Preoperative anteroposterior X-ray view. (b) Preoperative lateral X-ray view.

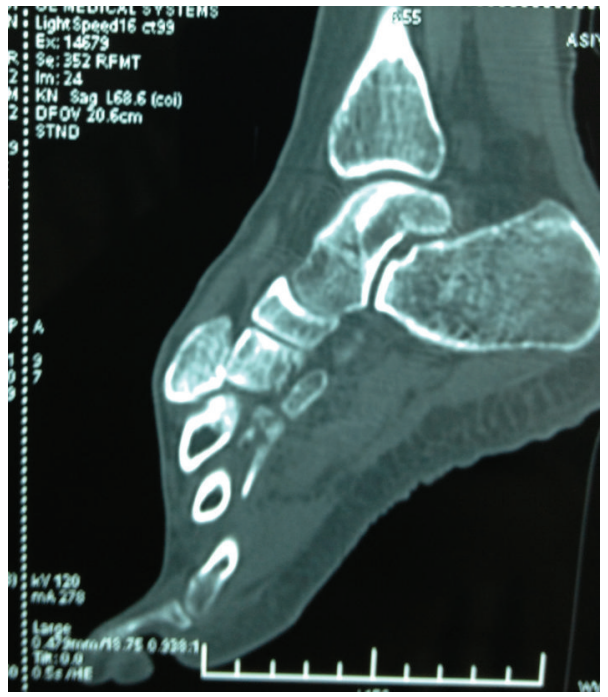

(a)

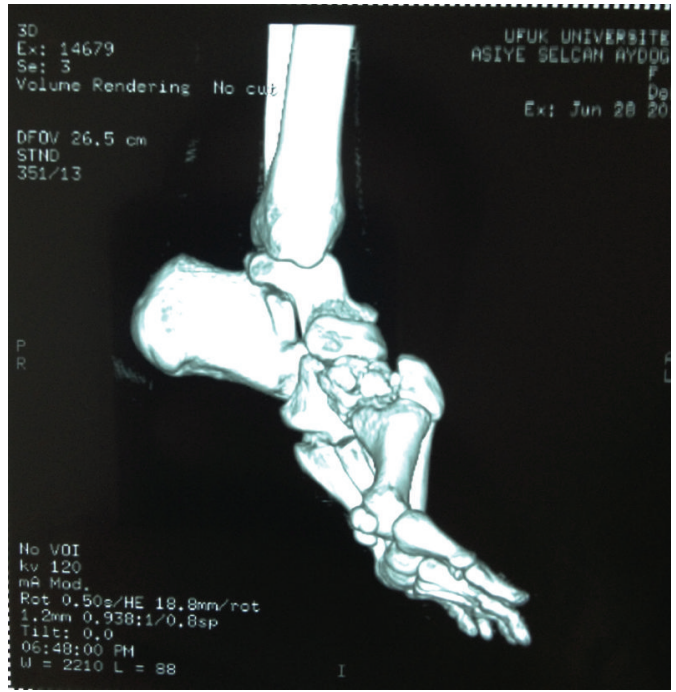

(b)

Figure 2: (a) Preoperative sagittal scan of computed tomography. (b) Preoperative three-dimensional reconstruction of computed tomography.

articulates with one third of the distal navicula proximally and its respective metatarsal distally [6]. The stability of these bones is achieved by the deep transverse, dorsal, and plantar ligaments. The plantar ligaments are strengthened by the tibialis posterior tendon. The intercuneiform joints are planartype joints that permit only gliding and rotation during pronation and supination movements $[7,8]$.

Isolated intermediate cuneiform dislocation was first described by Clark and Quint in 1933 [9]. In the literature, 13 cases of intermediate cuneiform dislocations have been associated with midfoot fractures: 10 were dorsal dislocations, and 3 were plantar [10]. Because the wedge shape is dorsally based, the intermediate cuneiform has a tendency to dislocate dorsally. Most intermediate cuneiform dislocations result from direct injury, but in some cases, indirect injury may cause the dislocation [11]. Nishi et al. performed an anatomic dissection to identify the mechanism of injury and the resultant pattern. According to their study, when the midfoot was under plantar flexion, the intermediate cuneiform was displaced dorsally, but when similar plantar flexion was applied under dorsal midfoot pressure, the intermediate cuneiform was prevented from dislocating dorsally [4]. In our opinion, dorsal shoe support helps maintain joint stability. Our patient was wearing high-heeled shoes with no dorsal support when she fell, and the intermediate cuneiform was therefore easily dislocated. Obtaining a detailed history of the mechanism of 


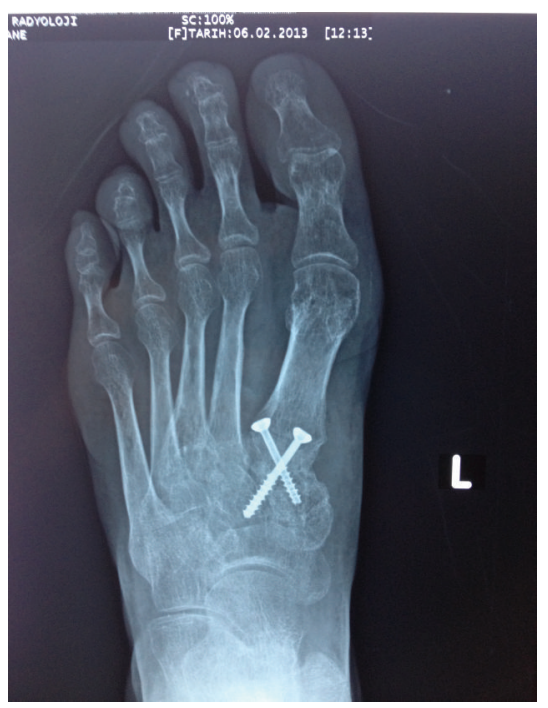

(a)

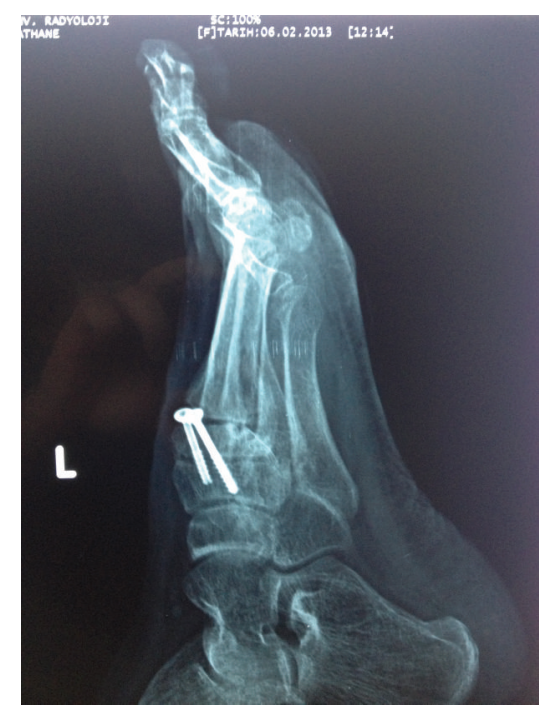

(b)

Figure 3: (a) Anteroposterior X-ray view 7 months after surgery. (b) Lateral X-ray view 7 months after surgery.

injury is important because the radiographic appearance of dislocation is confusing. The midtarsal foot anatomy is complicated with overlapping articulations that result in superimpositions on radiographical images [5]. In our case, we used computed tomography with three-dimensional reconstruction to determine the direction of dislocation and the displacement of the fracture to prevent overlooking another dislocation or fracture.

In this case, closed reduction under general anesthesia failed, and we had to reduce openly. Five of the 6 reported cases with intermediate cuneiform dislocation were treated by open reduction $[1,2]$. Although the failure rates of closed reduction are high, it should be attempted before open procedures. In conclusion, knowing the patient's choice of shoe is important for determining the mechanism of the injury. Immobilization and the avoidance of weight-bearing activity should continue for at least 6 weeks after surgery for successful treatment.

\section{Disclosure}

The authors of this paper required no funding for the creation of this study. Additionally, the authors have no financial disclosures to report. This paper has not been submitted to any other journal, and its text is original.

\section{References}

[1] D. Doshi, P. Prabhu, and A. Bhattacharjee, "Dorsal dislocation of the intermediate cuneiform with fracture of the lisfranc joint: a case report," Journal of Foot and Ankle Surgery, vol. 47, no. 1, pp. 60-62, 2008.

[2] R. Maitra and L. T. DeGnore, "Isolated dislocation of the middle cuneiform in a farmer: a case report and review of the literature," Foot and Ankle International, vol. 18, no. 11, pp. 735-738, 1997.
[3] M. Nashi and B. Banerjee, "Isolated plantar dislocation of the middle cuneiform - a case report," Injury, vol. 28, no. 9-10, pp. 704-706, 1997.

[4] H. Nishi, M. Takao, Y. Uchio, and N. Yamagami, "Isolated plantar dislocation of the intermediate cuneiform bone: a case report," Journal of Bone and Joint Surgery A, vol. 86, no. 8, pp. 1772-1777, 2004.

[5] F. Guler, A. B. Baz, A. Turan, O. Kose, and S. Akalin, "Isolated medial cuneiform fractures: report of two cases and review of the literature," Foot \& Ankle Specialist, vol. 4, no. 5, pp. 306-309, 2011.

[6] J. S. Early, "Cuneiform injuries," in Rockwood and Green's Fractures in Adults, C. A. Rockwood, D. P. Green, J. D. Heckman, and R. W. Bucholz, Eds., pp. 2199-2202, Lippincott Williams \& Wilkins, Philadelphia, Pa, USA, 2001.

[7] P. K. Aggarwal, S. Singh, and S. Kumar, "Isolated dorsal dislocation of the intermediate cunieform: a case report and review of the literature," Archives of Orthopaedic and Trauma Surgery, vol. 123, no. 5, pp. 252-253, 2003.

[8] H. Gray, Anatomy of the Human Body, Churchill Livingstone, Edinburgh, Scotland, 37th edition, 1989.

[9] D. F. Clark and H. A. Quint, "Dislocation of a single cuneiform bone," Journal of Bone and Joint Surgery A, vol. 15, pp. 237-239, 1933.

[10] M. Fujita, H. Yamamoto, K. Kariyama, and H. Yamakawa, "Isolated plantar dislocation of the middle cuneiform: a case report," The Journal of Orthopaedic Science, vol. 8, no. 6, pp. 875877, 2003.

[11] A. P. Aitken and D. Poulson, "Dislocations of the tarsometatarsal joint," The American Journal of Orthopedics, vol. 45, pp. 246-260, 1963. 


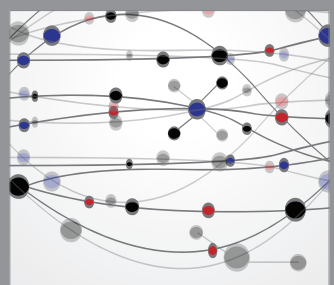

The Scientific World Journal
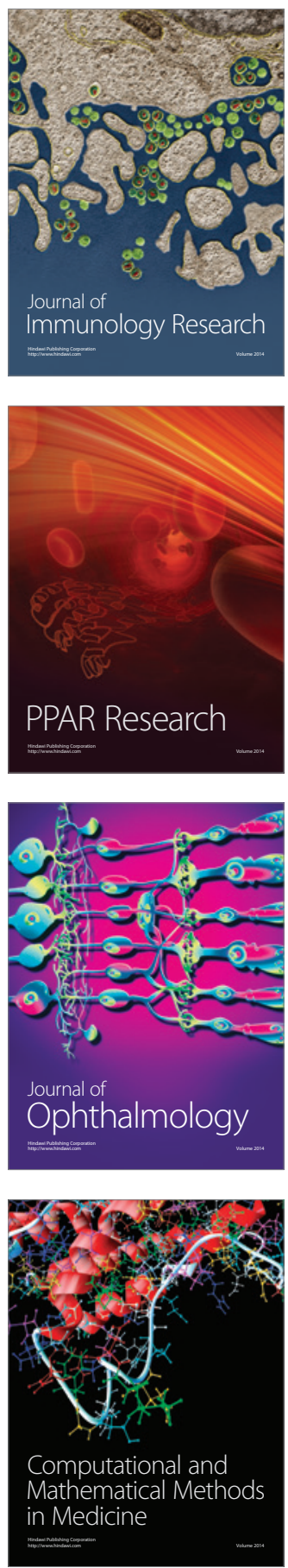

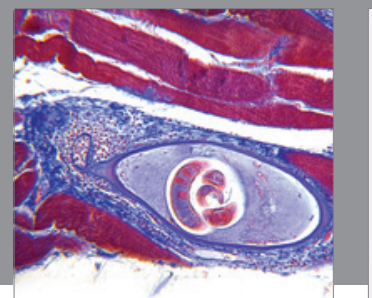

Gastroenterology

Research and Practice
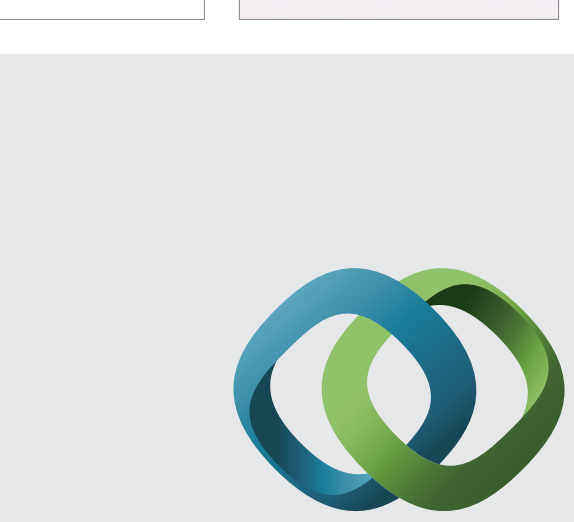

\section{Hindawi}

Submit your manuscripts at

http://www.hindawi.com
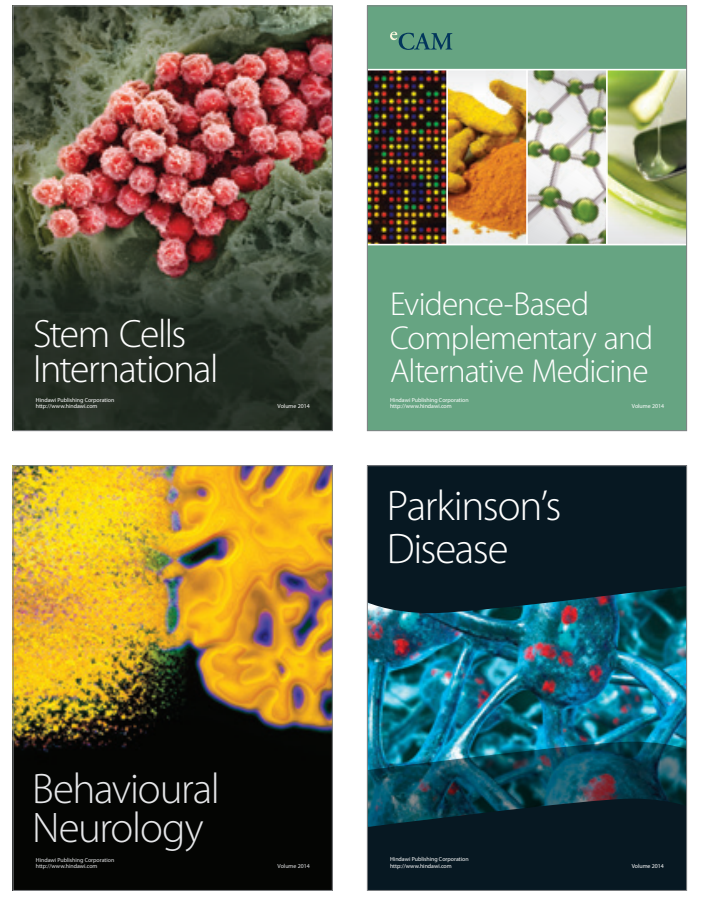
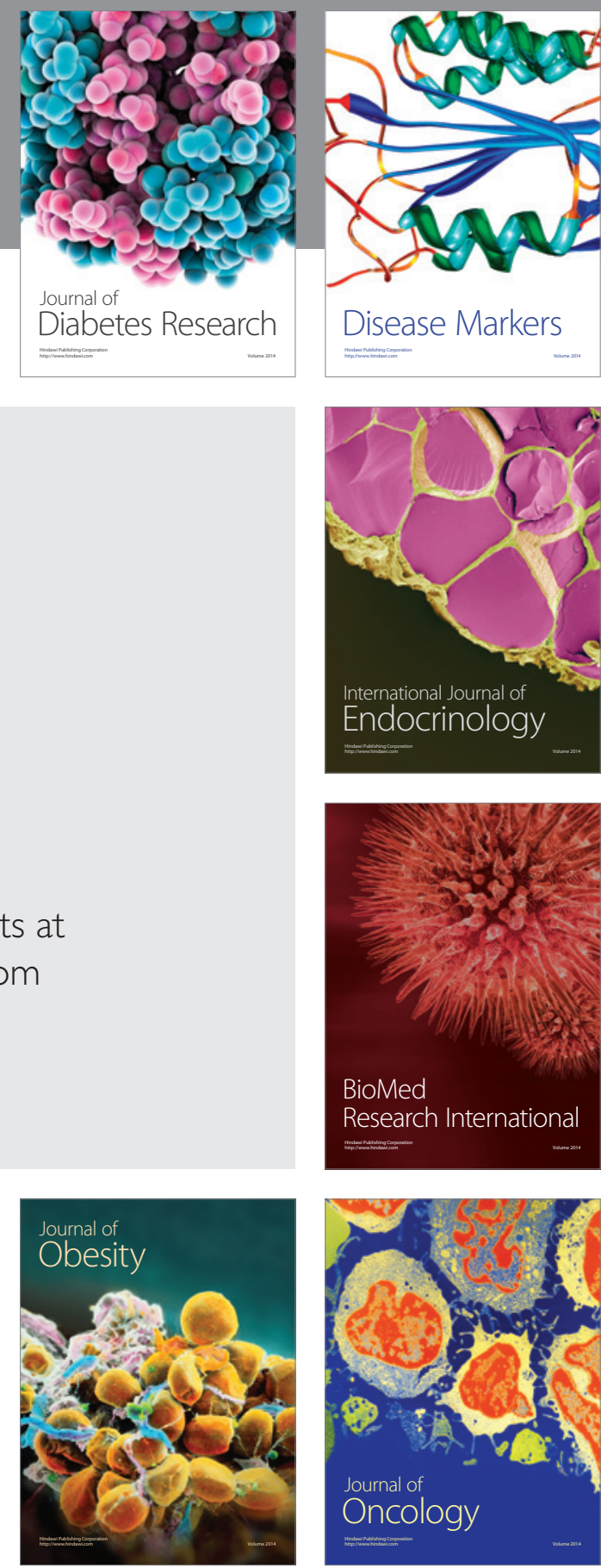

Disease Markers
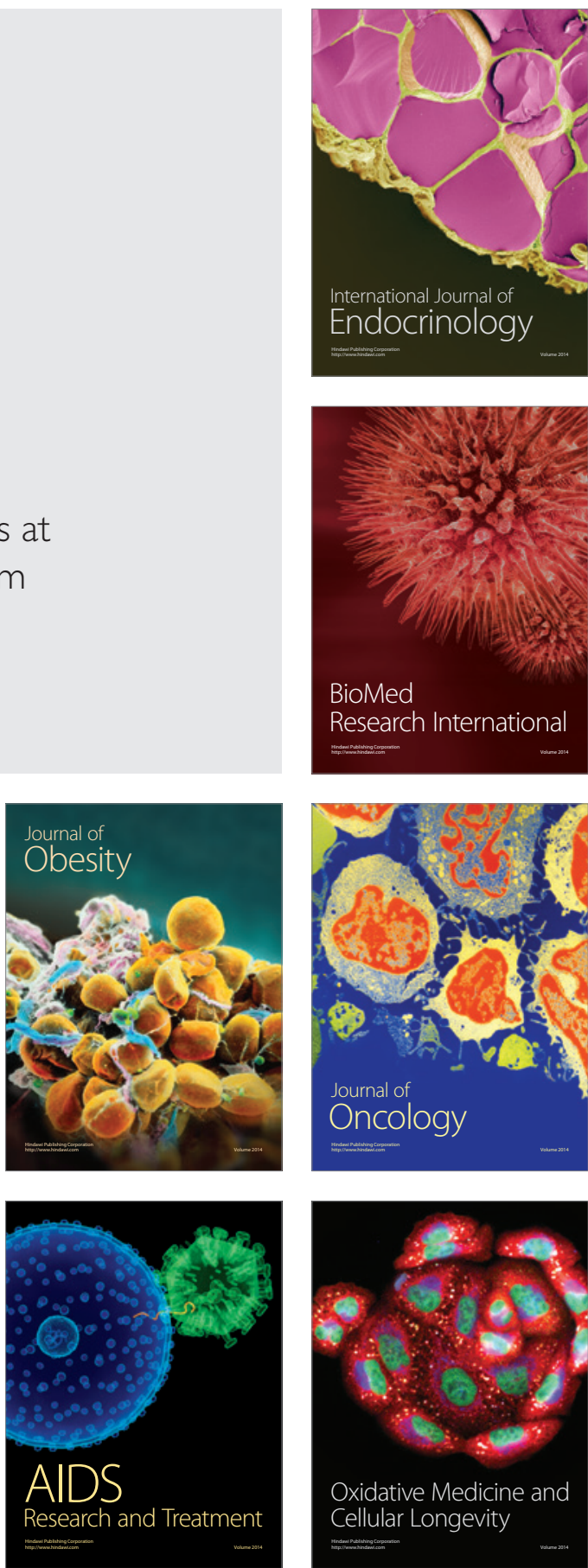